\title{
Review Article \\ Combination of Photodynamic Therapy with Radiotherapy for Cancer Treatment
}

\author{
Jing Xu, ${ }^{1}$ Jianqing Gao, ${ }^{2}$ and Qichun Wei ${ }^{1}$ \\ ${ }^{1}$ Department of Radiation Oncology, The Second Affiliated Hospital, Zhejiang University School of Medicine, Hangzhou 310009, China \\ ${ }^{2}$ Institute of Pharmaceutics, College of Pharmaceutical Sciences, Zhejiang University, Hangzhou 310009, China
}

Correspondence should be addressed to Qichun Wei; qichun_wei@zju.edu.cn

Received 28 January 2016; Revised 20 April 2016; Accepted 3 May 2016

Academic Editor: Peng Huang

Copyright (C) 2016 Jing Xu et al. This is an open access article distributed under the Creative Commons Attribution License, which permits unrestricted use, distribution, and reproduction in any medium, provided the original work is properly cited.

Photodynamic therapy (PDT) is a promising treatment modality for the management of malignant diseases. However, general acceptance of PDT has been hampered due to the limited tissue penetration of light and unavailability of suitable photosensitizers (PSs). The innovative combination of the conventional radiotherapy (RT) with PDT might reduce the unacceptable normal tissue toxicity while maintaining the desired tumor suppression effect. There have been a number of attempts to examine the interaction of PDT and RT; however, the previous results presented are ambiguous. The exact mechanisms for the variable responses of diverse panel of cell lines to the combination therapeutic regimen are still unclear. Novel ways are being explored to overcome the weaknesses of the conventional PDT and RT treatment regimen. The novel application to enable PDT of deep cancers is the utilization of scintillating nanoparticles as an intracellular light source for PDT activation. Upon simulation by X-rays, the nanoparticles emit scintillation or persistent luminescence to activate the PS to generate singlet oxygen. For future clinical applications, several questions are worthy of further elucidation, including the specific light dose, PS dose, radiation dose, the risk of complications, and the accurate time interval between administration of PDT and administration of RT.

\section{Introduction}

Radiation therapy (RT) has traditionally been one of the most common and efficient treatments of cancer and other diseases with ionizing radiation. RT causes lethal damage to disease cells by damaging the cellular DNA. Undifferentiated tumor cells are considered more susceptible to RT as they have a diminished ability to repair sublethal DNA damage [1]. A malignant cell's ability to repair radiation-induced DNA damage leads to a radioresistant phenotype. Besides, radiosensitivity also depends on the phase of the cell cycle at the time of irradiation. It is suggested that tumor cells in G1/S phases of the cell cycle are three times more radioresistant than those in G2/M phases [2].

The last several decades have witnessed substantial technological and methodological improvements of RT. It is worth noting that the advent of nanoscale technologies has encouraged the development of strategies to improve RT outcomes. For example, a peptide (HVGGSSV) bound liposome-encapsulated doxorubicin can bind specifically to irradiated tumors; hence, nanoparticle bound chemotherapy can be "guided" to tumor targets [3]. Besides, nanoparticle bound chemotherapy such as liposomal doxorubicin and nano-albumin bound paclitaxel may greatly reduce RT doses required to achieve tumor control [4]. The use of high-atomic number element nanoparticles as radiosensitizers is another extremely attractive approach on the horizon [5]. However, RT is still limited by unacceptable normal tissue toxicity in some cases. The dose is a critical issue because it increases the likelihood of damaging normal tissue surrounding the cancer. The ultimate goal of RT is to increase dose to the tumor while sparing normal tissue, thus improving the therapeutic ratio and reducing the side effects [6]. Nowadays, improving the clinical benefit of RT has been a long-standing goal in clinical cancer research.

Photodynamic therapy (PDT) has emerged as a promising alternative clinical treatment for the management of malignant diseases [7]. PDT involves the administration of photosensitizers (PSs) followed by illumination of the tumor with a localized energy source to activate the specific PS. 


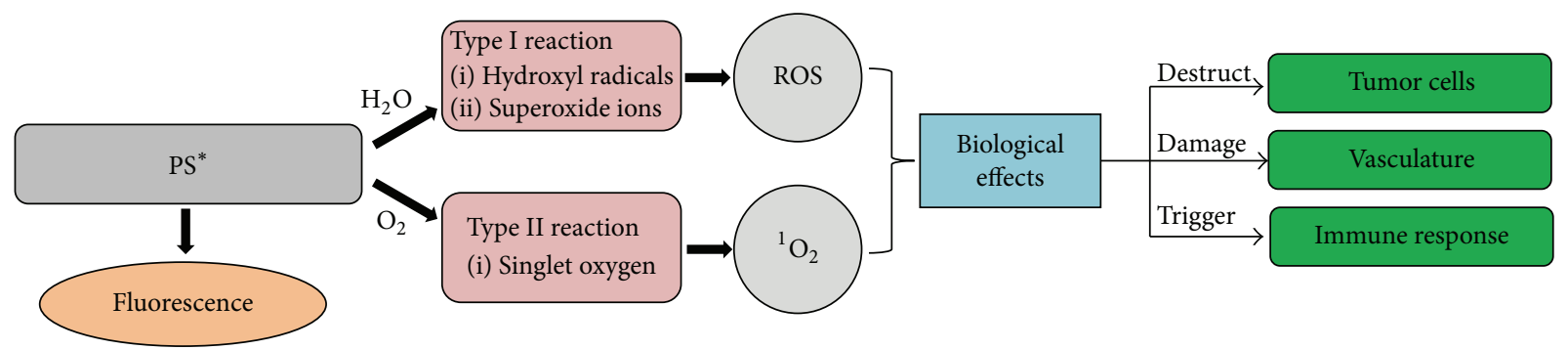

FIGURE 1: The overview of photodynamic reaction. PS*: active photosensitizer. ROS: reactive oxygen species.

Figure 1 illustrates several different pathways that lead to the biological effects when a PS is activated. Direct destruction of tumor cells, damage to the vasculature, and triggering of an antitumor immune response are three important cell death mechanisms in PDT [7]. The efficacy of PDT in the treatment of cancer is dependent on all these mechanisms and the relative contribution of each depends on the treatment regimen given [8]. PDT has attracted ever-growing attention because it combines two essentially harmless components (PS and visible light) to produce cytotoxic singlet oxygen that results in damage to cell membrane structures, microvascular ischaemia, and tissue necrosis [9]. Furthermore, due to the ability to preserve the anatomic and functional integrity of many organs, ease of application, selective targeting, and no drug resistance, PDT is a minimally invasive alternative for surgery or radiation [10]. Three parameters (light, PSs, and oxygen) determine the treatment outcome of PDT. Drug uptake and the dose of light depend on the tissue type; the level of oxygen is highly influenced by the initial tissue oxygenation and the rate of oxygen depletion, which changes as a result of PDT-induced hypoxia through vascular collapse [11]. Numerous clinical trials of PDT have been conducted and PDT is used with increasing frequency in a variety of cancers, such as bladder, skin, lung, esophagus, and cervix [12].

For the PDT to work properly, visible light must be effectively delivered to the PSs to excite them. However, most existing PSs are activated at the wavelength range 630$690 \mathrm{~nm}$, at which the tissue penetration depth of light is only 2-4 mm [13]. As a consequence, poor tissue penetrating ability of light limits PDT applications to treating superficial lesions (e.g., skin cancer) [14]. To address light penetration issues, long wavelength light sources are often used. Unfortunately, this can come as a tradeoff with excitation losses due to poor overlap with absorption spectra [15].

A wide variety of PSs have been developed and tested for PDT. Approved PDT drugs for oncological indications are listed in Table 1. First-generation PSs are hematoporphyrin, its derivative $(\mathrm{HpD})$, and the purified, commercially available Photofrin (Quadra Logic Technologies, Vancouver, Canada). Although the first-generation PSs have been shown to be efficacious in the treatment of many cancer types, these drugs are plagued with a uniformly high level of induced cutaneous phototoxicity and increased risk of sunburns. Phototoxic incidences of $20-40 \%$ have been reported during follow-up of patients having received Photofrin [16]. In addition, tumor size is another factor to determine the efficacy of PDT with Photofrin. It is reported that the response rate falls from $98 \%$ to $43 \%$ when the tumor is larger than $10 \mathrm{~mm}$ in diameter [17]. Recently, considerable efforts have been devoted to search for more tumor-selective photosensitizing agents with reduced side effects, called second-generation PSs. Most of the second-generation PSs are cyclic tetrapyrroles, including porphyrin and chlorin derivatives, phthalocyanines, and bacteriochlorins (Figure 2). While second-generation sensitizers are often totally synthetic, with perhaps a greater ability to generate singlet oxygen, they also have severe drawbacks. One is that they can potentially generate significant pain during therapy; another is that actually they are so active that even dim light (60-watt bulb) can lead to severe skin photosensitivity [18]. In searching for new and better PSs, the following requirements have been generally accepted as criteria for suitable PS agents, including chemical purity and known composition, lack of dark toxicity, preferential retainment to the target tissue, rapid pharmacokinetics, high quantum yield for photochemical event, minimal skin photosensitivity, commercial availability, and regulatory approval for clinical application. Although several commercially available and regulatory-approved PSs have achieved clinically successful outcomes, no PSs excellently approach these characteristics [19]. Generally, the significant limitations of PDT include inability to deliver adequate light doses to the target and unavailability of suitable PSs. It is suggested that improvement of more efficient PSs can be aided by synthesis of the compounds with strong absorption bands in the nearinfrared (NIR) region of the spectrum [20].

\section{Effects of the Combined Treatment}

As malignant diseases continue to plague humanity, significant effort should be invested in establishing optimal combination modalities for improving the efficacy and therapeutic effects of anticancer therapy. It is suggested that some PDTresistant tumor cells are more sensitive to ionizing radiation and some radiation-resistant tumor cells are sensitive to PDT [21]. Meanwhile, it is well known that photocytotoxic damage is caused by the singlet oxygen acting on the cell membranes, while the principal target for ionizing radiation-induced cell killing is nuclear DNA [22]. Therefore, PDT and RT induce damage on different targets. A synergism between them in killing cells might improve tumor control [23]. Taking into account these findings, it appears of particular interest to 
TABLE 1: Photosensitizers approved in human diseases.

\begin{tabular}{|c|c|c|}
\hline Indications & Photosensitizer & Country \\
\hline Actinic keratosis & 5- $A L A$ & US, EU \\
\hline $\begin{array}{l}\text { Basal cell } \\
\text { carcinoma }\end{array}$ & 5-Aminolevulinate & US, EU \\
\hline Barrett's dysplasia & Porfimer sodium & $\begin{array}{l}\text { US, Canada, EU, and } \\
\text { UK }\end{array}$ \\
\hline Cervical cancer & Porfimer sodium & Japan \\
\hline $\begin{array}{l}\text { Endobronchial } \\
\text { cancer }\end{array}$ & Porfimer sodium & $\begin{array}{l}\text { Canada, most EU } \\
\text { countries, Japan, and } \\
\text { US }\end{array}$ \\
\hline Esophageal cancer & Porfimer sodium & $\begin{array}{l}\text { Canada, most EU } \\
\text { countries, Japan, and } \\
\text { US }\end{array}$ \\
\hline Gastric cancer & Porfimer sodium & Japan \\
\hline $\begin{array}{l}\text { Papillary bladder } \\
\text { cancer }\end{array}$ & Porfimer sodium & Canada \\
\hline $\begin{array}{l}\text { Head and neck } \\
\text { cancer }\end{array}$ & Foscan & $\begin{array}{l}\text { EU, Norway, and } \\
\text { Iceland }\end{array}$ \\
\hline $\begin{array}{l}\text { Age-related } \\
\text { macular } \\
\text { degeneration }\end{array}$ & Verteporfin & $\begin{array}{l}\text { Canada, most EU } \\
\text { countries, Japan, and } \\
\text { US }\end{array}$ \\
\hline
\end{tabular}
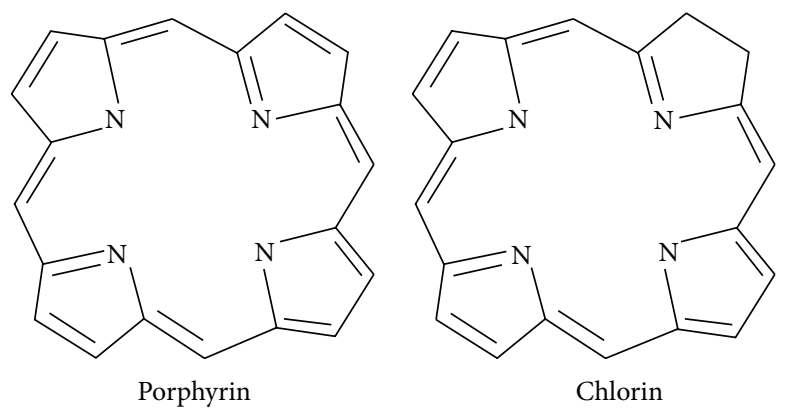

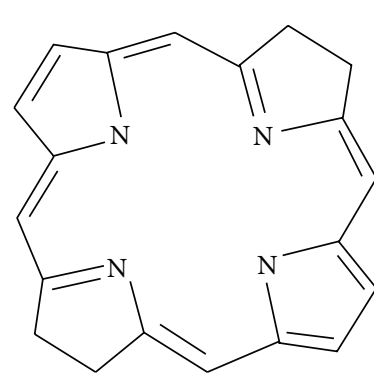

Bacteriochlorin

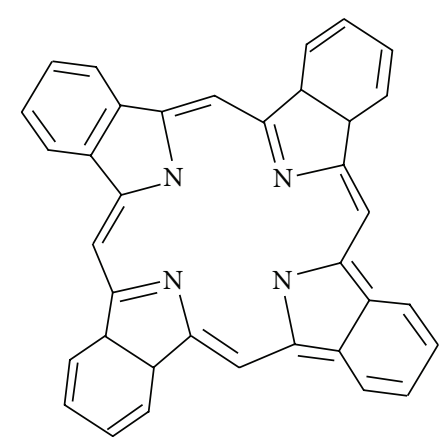

Phthalocyanine
FIGURE 2: Chemical structures of main photosensitizers used in PDT.

evaluate the antitumor effect of the innovative combination therapy. It is of importance since the potential damage caused by RT to normal issues can be minimized if the ionizing radiation dose can be reduced while maintaining the desired tumor suppression effect.

For this purpose, the effects of indocyanine green (ICG) as a sensitizer following combined treatment with RT and
PDT in MCF-7 human breast cancer cells were evaluated by Montazerabadi et al. The results showed that a combination of low doses ( $50 \mu \mathrm{M}$ ICG, $60 \mathrm{~J} / \mathrm{cm}^{2}$ light, and 4 Gy X-ray radiation) astonishingly resulted in the death of cancer cells and reduced the percentage of viable cancer cells to be $3.42 \%$. The adverse effects of PDT in combination with RT were partially abated, without a reduction in the efficacy of treatment [21]. Similar results were obtained using mitoxantrone (MX) as a sensitizer following combined treatment with RT and PDT in the MCF-7 cells by the same work team. It appeared that the combination modality had a strong cytotoxic effect and dose-dependent response on MCF-7 cells. Furthermore, the combination of PDT at $10 \mathrm{~J} / \mathrm{Cm}^{2}$ and $4 \mathrm{~Gy}$ of $\mathrm{X}$-ray radiation strongly reduced the percentage of viable cancer cells to $2.4 \pm$ 1.15 [10]. In an agreement with previous studies, Ghoodarzi et al. have shown consistent results recently [24]. Summarizing the data, this new type of combined treatment may open a new door for cancer treatment.

There have been a number of attempts to examine the interaction of PDT and RT; however, the previous results presented were indeterminate. In some previous reports, the cumulative effects of the combination therapy with PDT and ionizing radiation were purely additive and did not show any synergistic effect $[25,26]$. However, evidence of a synergistic interaction has been shown in other investigations. Bowen's disease (BD) (also known as "squamous cell carcinoma in situ") is a neoplastic skin disease that is considered as either an early stage or intraepidermal form of squamous cell carcinoma. In addition to surgical resection, PDT and RT were effective for the treatment of BD [27]. Particularly for the topical 5-aminolevulinic acid- (ALA-) $\mathrm{PDT}$, which was widely used for treating $\mathrm{BD}$, the cure rate was estimated to be $80-100 \%$ [28]. A combination of ALAPDT and RT was administered for the treatment of $\mathrm{BD}$, to overcome the limitations of conventional therapies, such as a relatively high recurrence rate, tumor cell persistence, and nonhealing ulcers, especially in the lower extremities [29]. It was promising that the combination therapy improved the effectiveness of ALA-PDT against BD and the synergetic effect of the therapy reduced the irradiation dose without causing any skin side effect [30]. In addition, it was also reported that the combined modality treatment by PDT with verteporfin and RT showed a significant enhancement in cytotoxicity compared with either PDT or RT alone [31].

\section{Mechanisms}

The exact mechanisms for the conflicting results of the combination therapy were unclear. Prinsze et al. [32] suggested that the synergistic interactions could be explained by the cell line differences in the sensitivity to PDT-induced inhibition of DNA repair. PDT resulted in inhibition of DNA repair in some cell lines, whereas in others DNA repair was insensitive to PDT. Furthermore, the dose and the time between the administration of the PS and tumor irradiation played an important role in the interaction of PDT and RT. Wang et al. [33] demonstrated that approximately half of the radiationinduced damage was repaired in the first $5 \mathrm{~min}$, whereas the remainder was repaired within $30 \mathrm{~min}$. This was totally 


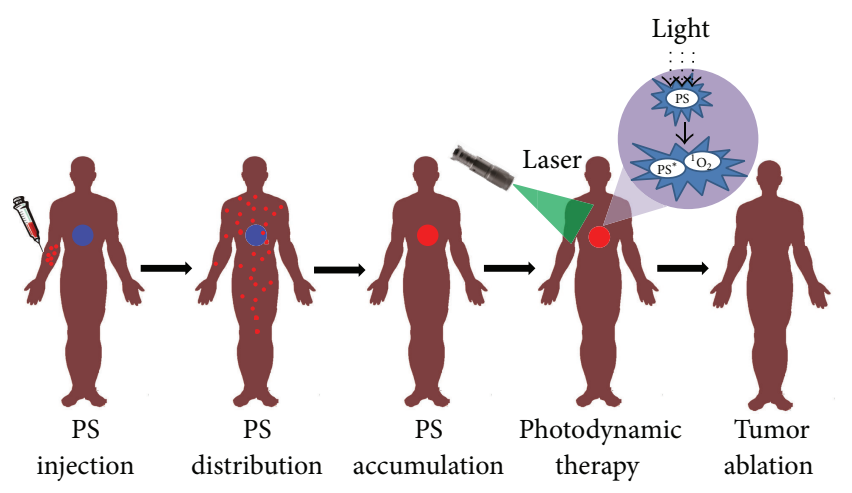

FIgURE 3: Clinical photodynamic therapy. ${ }^{1} \mathrm{O}_{2}$ : singlet oxygen; PS: photosensitizer; and $\mathrm{PS}^{*}$ : active photosensitizer.

consistent with the finding that the percentage of viability potentially reduced when hematoporphyrin treated cells were irradiated simultaneously with light and X-rays [34].

\section{In Vivo Studies}

Cell culture and animal studies indicated a possible synergistic effect of combining PDT with RT. Unfortunately, there had been only limited investigation using in vivo models. PDT in vivo starts with application of PS systemically or topically. Then PS selectively accumulates in the neoplastic lesions and a light of appropriate wavelength activates the PS and generates ${ }^{1} \mathrm{O}_{2}$, leading to tumor ablation (Figure 3) [35]. From a literature, thirty-two patients with histologically proven non-small-cell bronchogenic tumor and bulky endobronchial tumor were treated using a combination of PDT (Photofrin, $2 \mathrm{mg} / \mathrm{kg}$ ) and brachytherapy. Six weeks after PDT, brachytherapy was applied with five fractions of $4 \mathrm{~Gy}$ at weekly intervals. All patients were alive after a mean of 24 months. The combined treatment regimen failed to eradicate the tumor in only one patient and distant metastases developed in two of the six patients. It was concluded that combined treatment with PDT and brachytherapy was safe and had excellent therapeutic efficacy [36]. Kusuzaki et al. investigated the usefulness of a unique modality of PDT using acridine orange (AO-PDT), including intralesional or partially marginal tumor excision and fluorescence-microscopic curettage using the fluorovisualization effect, with/without 5 Gy radiation, in 10 patients with musculoskeletal sarcoma. The follow-up of the patients ranged from 24 to 48 months. The results revealed that all the patients (AO-PDT alone: 5, AO-PDT with 5 Gy radiation: 5) are alive. The recurrence rate was $10 \%$, which is almost the same as that after wide tumor resection. None of the 5 patients who received AO-PDT with radiation developed local tumor recurrence. The limb function in all the patients, except for one, recovered to the level before surgery. None of the patients clinically showed any severe complications, such as skin hypersensitivity to light. Hence, the combination therapy of AO-PDT and lowdose RT may be a promising new limb salvage modality to achieve satisfactory improvement of limb function in patients with malignant bone and soft tissue tumors [37].
However, an unacceptable high rate of complications of PDT was described in 3 patients with carcinoma of the upper aerodigestive tract. Two patients received prior treatment with external beam irradiation (55.8 and $50.4 \mathrm{~Gy})$ and intraluminal brachytherapy (IB) (12 Gy and $35 \mathrm{~Gy}$ at $1 \mathrm{~cm}$ ), followed by PDT for treatment of recurrence. One patient was treated with external beam irradiation (60 Gy) followed by IB $(36.1 \mathrm{~Gy}$ at $1 \mathrm{~cm})$ and then received PDT for recurrence. Unfortunately, one patient had a tracheoesophageal fistula requiring stent placement, another developed quadriplegia due to an epidural abscess, and a third had fatal pulmonary arterial hemorrhage. Loss of a critical number of cells and changes in the biochemical microenvironment, which may play a role in the development of late tissue changes and additive toxicity, were the possible mechanisms of the toxicity of the combined therapy [38]. One thing has to be mentioned; all these three patients received higher radiation dose than usual, so it is hard to comment on the relationship between the severe complications and the application of PDT. The results are to some extent ambiguous; the degree of interaction appears to depend on numerous parameters, including the type of pathology, the dose and dose rate of both ionizing radiation and light, and the sequence and timing of treatments. This new therapeutic avenue for tumor therapy merits further controlled trials for application in human in future.

\section{Promising Emerging Approaches}

Novel ways were being explored to overcome the inadequacies of the conventional PDT and RT treatment. Nanoparticles are multifunctional molecules with diameters varying between 1 and $1000 \mathrm{~nm}$ [39]. In the nanoparticle-based approach, the sensitizer is encapsulated or immobilized on the nanoparticle surface using covalent/noncovalent interactions. The use of nanoparticles as carriers of PS might bring challenging new solutions to the PDT as nanomaterials can satisfy all the requirements for an ideal PDT agent [40]. The advantage of this approach is a more direct and specific localization of the PS to the tumor site with increased efficiency and selectivity [41]. The promising emerging approach of using nanoparticles to enable the combination of PDT with RT for deep cancer treatment was proposed by Chen and Zhang in 2006 [42]. Under this concept, luminescent nanoparticles were utilized for the delivery of PS such as porphyrin. Upon simulation by X-rays, the nanoparticles emit scintillation or persistent luminescence to activate the PS to generate singlet oxygen. The novel strategy described in this study involves the use of in vivo luminescent nanoparticles so that an external light source is not necessary to activate the photosensitizing agent within tumors. Moreover, highenergy beams such as X-rays can penetrate deep tissue easily; therefore, after the PDT activation by X-ray, it will be feasible for deep cancer treatment (Figure 4).

The use of X-ray induced luminescence nanoparticles for PDT activation, permitting treatment of deeper tumors, has become a hot topic for cancer treatment. X-ray-activated PDT can combine with RT for treatment enhancement was confirmed by the following examples. $\mathrm{LaF}_{3}: \mathrm{Ce}^{3+}$ luminescent 


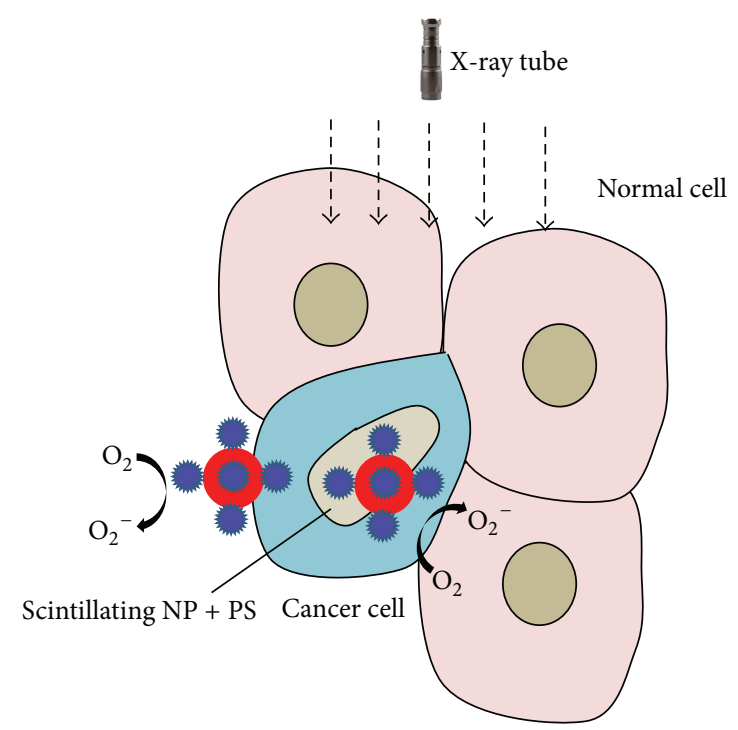

FIGURE 4: Schematic of combination therapy of radiotherapy and photodynamic therapy. Ionizing radiation is used to excite scintillating nanoparticles, which may be located deep within tissue. The nanoparticles transfer energy to the attached photosensitizer molecules, killing cells by the same mechanism as photodynamic therapy.

nanoparticles were synthesized by a wet-chemistry method in dimethyl sulfoxide (DMSO), and their application as an intracellular light source for PDT activation was demonstrated. Emission spectrum of the $\mathrm{Ce}^{3+}$-doped lanthanum (III) fluoride $\left(\mathrm{LaF}_{3}: \mathrm{Ce}^{3+}\right)$ is effectively overlapped with the absorption of protoporphyrin IX (PPIX). Upon irradiation with X-rays $(90 \mathrm{kV}), \mathrm{LaF}_{3}: \mathrm{Ce}^{3+}$ and PPIX nanocomposites induced oxidative stress, mitochondrial damage, and DNA fragmentation on prostate cancer cells (PC3) [43]. Meanwhile, copper-cysteamine complex ( $\mathrm{Cu}-\mathrm{Cy})$ nanoparticles were exploited as a new structured photosensitizer that could be activated both by light and by X-ray. The $\mathrm{Cu}-\mathrm{Cy}$ nanoparticles could be effectively activated by X-rays (1 $8 \mathrm{~Gy}$ ) to produce singlet oxygen. In vitro and in vivo study on human breast cancer cells (MCF-7) have demonstrated significant cell destruction using $\mathrm{Cu}-\mathrm{Cy}$ nanoparticles under $\mathrm{X}$ ray activation. The $\mathrm{Cu}-\mathrm{Cy}$ nanoparticles have several unique characteristics, such as low cytotoxicity, easiness of synthesis, and fabrication at the nanoscale to increase water solubility and cellular uptake. Overall, a novel and promising X-ray activated $\mathrm{Cu}-\mathrm{Cy}$ PS could enable PDT for both shallow and deep cancers [44].

Several possible drawbacks still contribute to the unsatisfying efficacy concerning the use of X-ray excited PDT. PSs used are usually porphyrins or relevant derivatives which possess maximal absorption around $400 \mathrm{~nm}$. Lanthanidedoped nanoparticles often exhibit strong emission between 450 and $600 \mathrm{~nm}$, which could not efficiently excite the absorption band of the attached PS to induce an efficient singlet oxygen generation. Second, the methods for the construction process of nanocomposites are usually complicated and the luminescence efficiency of scintillating particles will be attenuated during the conjugation procedures [45]. Moreover, more than 2 Gy X-ray irradiation was usually needed to achieve the desired treatment effects in the aforementioned approaches, a dose that is comparable to or even higher than daily dose in the conventionally fractioned RT in clinic. The novel methods for further enhancing the efficiency of the nanoparticle-based combined therapy are emerging. A novel hybrid nanosystem was proposed based on biocompatible inorganic $\mathrm{SiC} / \mathrm{SiO}_{x}$ core/shell nanowires conjugated via click-chemistry procedures with an organic PS, a tetracarboxyphenyl porphyrin derivative. The system is an efficient source of ${ }^{1} \mathrm{O}_{2}$ for cell oxidative stress when irradiated with $6 \mathrm{MV}$ X-rays even in extremely low-dose irradiation $(0.4 \mathrm{~Gy})$ [46]. The integrated nanosystem, which consisted of a core made of $\mathrm{SrAl}_{2} \mathrm{O}_{4}: \mathrm{Eu}^{2+}(\mathrm{SAO})$ and a mesoporous silica coating loaded with PS (merocyanine 540), was used for X-ray-to-visible conversion to activate the PS. Upon low X-ray irradiation ( $0.5 \mathrm{~Gy})$, efficient tumor shrinkage was caused while leaving the normal tissues unaffected in murine U87MG xenograft models [47]. Mesoporous $\mathrm{LaF}_{3}: \mathrm{Tb}$ scintillating nanoparticles (ScNPs) were successfully synthesized by a facile hydrothermal process. The main absorption of watersoluble PS molecules named Rose Bengal (RB) at $549 \mathrm{~nm}$ overlaps exactly with the main emission band of $\mathrm{LaF}_{3}: \mathrm{Tb}$ ScNPs at $544 \mathrm{~nm}$. The Försterresonance energy transfer (FRET) efficiency between ScNPs and RB was calculated to be 85\%. Overall, the $\mathrm{LaF}_{3}$ :Tb-RB FRET system possesses several advantages including well-defined nanostructure, optimized scintillating luminescence, admired spectrum overlap, simple drug loading approach, good water solubility, and ultra colloidal stability. Hence, this system shows great potential to be applied in X-ray stimulated PDT for deep-seated tumors in the future [45]. The exploration of luminescence-activated PDT employing afterglow luminescence combined with scintillation luminescence is a novel concept for further enhancement of the PDT and RT [48]. The afterglow can be charged by irradiation for a few seconds. In this case, even if the $\mathrm{X}$ ray source is off, the PDT is still active because of the long afterglow from the selected nanoparticles. This will provide an efficient, simple, convenient, and inexpensive in vivo light source for PDT treatment. It is demonstrated that 2-deoxy-2$\left[{ }^{18} \mathrm{~F}\right]$ fluoro-D-glucose $\left({ }^{18} \mathrm{FDG}\right)$, which is a metabolic activitybased positron emission tomography (PET) probe, could be used as an alternative light source for photoactivation. A promising way to address depth can be accomplished noninvasively with Cherenkov radiation, by moving the light source colocalizing with photoactive agents in vivo [15].

These approaches are expected to find wide application in the clinic, especially for tumors that are resistant to RT. However, appropriate particle dimension and optimized surface features are needed for practical applications, since the size of particles should be large enough to prevent rapid leakage in blood capillaries and small enough to escape the capture of macrophages in the reticuloendothelial system (RES). Furthermore, combination nanomedicines can be further modified with antibody or other tumor targeting molecules to increase tumor deposition and reduce toxicity in adjacent normal tissue [49]. 


\section{Conclusions and Outlook}

In summary, we have reviewed the innovative combination therapy of RT and PDT, which is utilized to overcome the weaknesses of the conventional RT and PDT treatment regimen. Novel PS and light sources are being developed; moreover, impressive progress in understanding of tumor cell biology raises hopes for further improvements in this promising combined method of cancer treatment. The effects of combined RT and PDT have currently been studied primarily inin vitro systems consisting of monolayer cell cultures. For future clinical applications, one of the challenging issues is to ensure that light dose can be adjusted according to the size and depth of the tumor within the body. PS dose can be adjusted according to the sex, weight, and age of the patients who are likely to receive RT and PDT concurrently. The other challenging issue is to determine what radiation dose is sufficient in vivo to generate enough light for PDT and the accurate time interval between administration of PDT and administration of RT. Lastly, although data on the complications of PDT as de novo treatment is available in the literature, there is little information on toxicity in PDT patients who have been treated with RT [50]. The toxicity of the combined therapy in patients should be further identified in future.

\section{Competing Interests}

The authors declare that there is no conflict of interests regarding the publication of this paper.

\section{References}

[1] I. Postiglione, A. Chiaviello, and G. Palumbo, "Enhancing photodynamyc therapy efficacy by combination therapy: dated, current and oncoming strategies," Cancers, vol. 3, no. 2, pp. 2597-2629, 2011.

[2] L. Wang, W. Yang, P. Read, J. Larner, and K. Sheng, "Tumor cell apoptosis induced by nanoparticle conjugate in combination with radiation therapy," Nanotechnology, vol. 21, no. 47, Article ID 475103, 2010.

[3] A. Lowery, H. Onishko, D. E. Hallahan, and Z. Han, “Tumortargeted delivery of liposome-encapsulated doxorubicin by use of a peptide that selectively binds to irradiated tumors," Journal of Controlled Release, vol. 150, no. 1, pp. 117-124, 2011.

[4] S. Revannasiddaiah and S. P. Susheela, "Chemically enhanced radiotherapy: visions for the future," Annals of Translational Medicine, vol. 4, no. 3, article 52, 2016.

[5] J. A. Coulter, W. B. Hyland, J. Nicol, and F. J. Currell, "Radiosensitising nanoparticles as novel cancer therapeutics-Pipe dream or realistic prospect?" Clinical Oncology, vol. 25, no. 10, pp. 593603, 2013.

[6] P. Tsiamas, B. Liu, F. Cifter et al., "Impact of beam quality on megavoltage radiotherapy treatment techniques utilizing gold nanoparticles for dose enhancement," Physics in Medicine and Biology, vol. 58, no. 3, pp. 451-464, 2013.

[7] M. Olivo, R. Bhuvaneswari, S. S. Lucky, N. Dendukuri, and P. S.-P. Thong, "Targeted therapy of cancer using photodynamic therapy in combination with multi-faceted anti-tumor modalities," Pharmaceuticals, vol. 3, no. 5, pp. 1507-1529, 2010.
[8] B. W. Henderson, S. O. Gollnick, J. W. Snyder et al., "Choice of oxygen-conserving treatment regimen determines the inflammatory response and outcome of photodynamic therapy of tumors," Cancer Research, vol. 64, no. 6, pp. 2120-2126, 2004.

[9] Y.-Y. Huang, S. K. Sharma, T. Dai et al., "Can nanotechnology potentiate photodynamic therapy?" Nanotechnology Reviews, vol. 1, no. 2, pp. 111-146, 2012.

[10] A. Sazgarnia, A. R. Montazerabadi, M. H. Bahreyni-Toosi, A. Ahmadi, and A. Aledavood, "In vitro survival of MCF-7 breast cancer cells following combined treatment with ionizing radiation and mitoxantrone-mediated photodynamic therapy," Photodiagnosis and Photodynamic Therapy, vol. 10, no. 1, pp. 7278, 2013.

[11] T. C. Zhu, E. I. Parsai, and C. G. Orton, "PDT is better than alternative therapies such as brachytherapy, electron beams, or low-energy X rays for the treatment of skin cancers," Medical Physics, vol. 38, no. 3, pp. 1133-1135, 2011.

[12] J.-O. Yoo and K.-S. Ha, "New insights into the mechanisms for photodynamic therapy-induced cancer cell death," International Review of Cell and Molecular Biology, vol. 295, pp. 139-174, 2012.

[13] P. N. Prasad, "Bioimaging: principles and techniques," in Introduction to Biophotonics, pp. 203-254, 2003.

[14] K. Kalka, H. Merk, and H. Mukhtar, "Photodynamic therapy in dermatology," Journal of the American Academy of Dermatology, vol. 42, no. 3, pp. 389-413, 2000.

[15] C. Ran, Z. Zhang, J. Hooker, and A. Moore, "In vivo photoactivation without 'light': use of cherenkov radiation to overcome the penetration limit of light," Molecular Imaging and Biology, vol. 14, no. 2, pp. 156-162, 2012.

[16] T. J. Dougherty, M. T. Cooper, and T. S. Mang, "Cutaneous phototoxic occurrences in patients receiving photofrin ${ }^{\circledR,}$, Lasers in Surgery and Medicine, vol. 10, no. 5, pp. 485-488, 1990.

[17] K. Furuse, M. Fukuoka, H. Kato et al., "A prospective phase II study on photodynamic therapy with photofrin II for centrally located early-stage lung cancer," Journal of Clinical Oncology, vol. 11, no. 10, pp. 1852-1857, 1993.

[18] R. R. Allison, G. H. Downie, R. Cuenca, X.-H. Hu, C. J. H. Childs, and C. H. Sibata, "Photosensitizers in clinical PDT," Photodiagnosis and Photodynamic Therapy, vol. 1, no. 1, pp. 2742, 2004.

[19] R. R. Allison, "Photodynamic therapy: oncologic horizons," Future Oncology, vol. 10, no. 1, pp. 123-124, 2014.

[20] L. Benov, "Photodynamic therapy: current status and future directions," Medical Principles and Practice, vol. 24, supplement 1, pp. 14-28, 2015.

[21] A. R. Montazerabadi, A. Sazgarnia, M. H. Bahreyni-Toosi, A. Ahmadi, and A. Aledavood, "The effects of combined treatment with ionizing radiation and indocyanine greenmediated photodynamic therapy on breast cancer cells," Journal of Photochemistry and Photobiology B: Biology, vol. 109, pp. 4249, 2012.

[22] A. Colasanti, A. Kisslinger, M. Quarto, and P. Riccio, "Combined effects of radiotherapy and photodynamic therapy on an in vitro human prostate model," Acta Biochimica Polonica, vol. 51, no. 4, pp. 1039-1046, 2004.

[23] N. Ramakrishnan, M. E. Clay, L. R. Friedman, A. R. Antunez, and N. L. Oleinick, "Post-treatment interactions of photodynamic and radiation-induced cytotoxic lesions," Photochemistry and Photobiology, vol. 52, no. 3, pp. 555-559, 1990. 
[24] R. Ghoodarzi, V. Changizi, A. R. Montazerabadi, and N. Eyvazzadaeh, "Assessing of integration of ionizing radiation with Radachlorin-PDT on MCF-7 breast cancer cell treatment," Lasers in Medical Science, vol. 31, no. 2, pp. 213-219, 2016.

[25] Z. Luksiene, A. Kalvelyte, and R. Supino, "On the combination of photodynamic therapy with ionizing radiation," Journal of Photochemistry and Photobiology B: Biology, vol. 52, no. 1-3, pp. 35-42, 1999.

[26] R. Allman, P. Cowburn, and M. Mason, "Effect of photodynamic therapy in combination with ionizing radiation on human squamous cell carcinoma cell lines of the head and neck," British Journal of Cancer, vol. 83, no. 5, pp. 655-661, 2000.

[27] P. Lehmann, "Methyl aminolaevulinate-photodynamic therapy: a review of clinical trials in the treatment of actinic keratoses and nonmelanoma skin cancer," British Journal of Dermatology, vol. 156, no. 5, pp. 793-801, 2007.

[28] N. H. Cox, D. J. Eedy, and C. A. Morton, "Guidelines for management of Bowen's disease," British Journal of Dermatology, vol. 141, no. 4, pp. 633-641, 1999.

[29] M. T. Dupree, R. A. Kiteley, K. Weismantle, R. Panos, and P. A. S. Johnstone, "Radiation therapy for bowen's disease: lessons for lesions of the lower extremity," Journal of the American Academy of Dermatology, vol. 45, no. 3, pp. 401-404, 2001.

[30] A. Nakano, D. Watanabe, Y. Akita, T. Kawamura, Y. Tamada, and Y. Matsumoto, "Treatment efficiency of combining photodynamic therapy and ionizing radiation for Bowens disease," Journal of the European Academy of Dermatology and Venereology, vol. 25, no. 4, pp. 475-478, 2011.

[31] B. W. Pogue, J. A. O'Hara, E. Demidenko et al., "Photodynamic therapy with verteporfin in the radiation-induced fibrosarcoma-1 tumor causes enhanced radiation sensitivity," Cancer Research, vol. 63, no. 5, pp. 1025-1033, 2003.

[32] C. Prinsze, L. C. Penning, T. M. A. R. Dubbelman, and J. VanSteveninck, "Interaction of photodynamic treatment and either hyperthermia or ionizing radiation and of ionizing radiation and hyperthermia with respect to cell killing of L929 fibroblasts, Chinese hamster ovary cells, and T24 human bladder carcinoma cells," Cancer Research, vol. 52, no. 1, pp. 117120, 1992.

[33] J. Wang, W. Hyun, K. Lamborn, and D. F. Deen, "Measurement of radiation-induced damage in human glioma cells with flow cytometry," Cancer Research, vol. 56, no. 1, pp. 154-157, 1996.

[34] G. Kavarnos, R. Nath, and P. Bongiorni, "Visible-light and $\mathrm{X}$ irradiations of Chinese hamster lung cells treated with hematoporphyrin derivative," Radiation Research, vol. 137, no. 2, pp. 196-201, 1994.

[35] P. Agostinis, K. Berg, K. A. Cengel et al., "Photodynamic therapy of cancer: an update," CA: A Cancer Journal for Clinicians, vol. 61, no. 4, pp. 250-281, 2011.

[36] L. Freitag, A. Ernst, M. Thomas, R. Prenzel, B. Wahlers, and H.-N. Macha, "Sequential photodynamic therapy (PDT) and high dose brachytherapy for endobronchial tumour control in patients with limited bronchogenic carcinoma," Thorax, vol. 59, no. 9, pp. 790-793, 2004.

[37] K. Kusuzaki, H. Murata, T. Matsubara et al., "Clinical trial of photodynamic therapy using acridine orange with/without low dose radiation as new limb salvage modality in musculoskeletal sarcomas," Anticancer Research, vol. 25, no. 2, pp. 1225-1235, 2005.

[38] N. J. Sanfilippo, A. Hsi, A. S. DeNittis et al., "Toxicity of photodynamic therapy after combined external beam radiotherapy and intraluminal brachytherapy for carcinoma of the upper aerodigestive tract," Lasers in Surgery and Medicine, vol. 28, no. 3, pp. 278-281, 2001.

[39] M. Firczuk, M. Winiarska, A. Szokalska et al., "Approaches to improve photodynamic therapy of cancer," Frontiers in Bioscience, vol. 16, no. 1, pp. 208-224, 2011.

[40] D. Bechet, P. Couleaud, C. Frochot, M.-L. Viriot, F. Guillemin, and M. Barberi-Heyob, "Nanoparticles as vehicles for delivery of photodynamic therapy agents," Trends in Biotechnology, vol. 26, no. 11, pp. 612-621, 2008.

[41] D. K. Chatterjee, L. S. Fong, and Y. Zhang, "Nanoparticles in photodynamic therapy: an emerging paradigm," Advanced Drug Delivery Reviews, vol. 60, no. 15, pp. 1627-1637, 2008.

[42] W. Chen and J. Zhang, "Using nanoparticles to enable simultaneous radiation and photodynamic therapies for cancer treatment," Journal of Nanoscience and Nanotechnology, vol. 6, no. 4, pp. 1159-1166, 2006.

[43] X. Zou, M. Yao, L. Ma et al., "X-ray-induced nanoparticle-based photodynamic therapy of cancer," Nanomedicine, vol. 9, no. 15, pp. 2339-2351, 2014.

[44] L. Ma, X. Zou, and W. Chen, "A new X-ray activated nanoparticle photosensitizer for cancer treatment," Journal of Biomedical Nanotechnology, vol. 10, no. 8, pp. 1501-1508, 2014.

[45] Y. Tang, J. Hu, A. H. Elmenoufy, and X. Yang, "Highly efficient FRET system capable of deep photodynamic therapy established on X-ray excited mesoporous $\mathrm{LaF}_{3}: \mathrm{Tb}$ scintillating nanoparticles," ACS Applied Materials and Interfaces, vol. 7, no. 22, pp. 12261-12269, 2015.

[46] F. Rossi, E. Bedogni, F. Bigi et al., "Porphyrin conjugated $\mathrm{SiO}_{\mathbf{x}}$ nanowires for X-ray-excited photodynamic therapy," Scientific Reports, vol. 5, article 7606, 2015.

[47] H. Chen, G. D. Wang, Y.-J. Chuang et al., "Nanoscintillatormediated X-ray inducible photodynamic therapy for in vivo cancer treatment," Nano Letters, vol. 15, no. 4, pp. 2249-2256, 2015.

[48] P. Juzenas, W. Chen, Y.-P. Sun et al., "Quantum dots and nanoparticles for photodynamic and radiation therapies of cancer," Advanced Drug Delivery Reviews, vol. 60, no. 15, pp. 1600-1614, 2008.

[49] C. He, C. Chan, R. R. Weichselbaum, G. F. Fleming, S. D. Yamada, and W. Lin, "Nanomedicine for combination therapy of cancer," EBioMedicine, vol. 2, no. 5, pp. 366-367, 2015.

[50] J. S. McCaughan Jr., E. C. Ellison, J. T. Guy et al., "Photodynamic therapy for esophageal malignancy: a prospective twelve-year study," Annals of Thoracic Surgery, vol. 62, no. 4, pp. 1005-1010, 1996. 

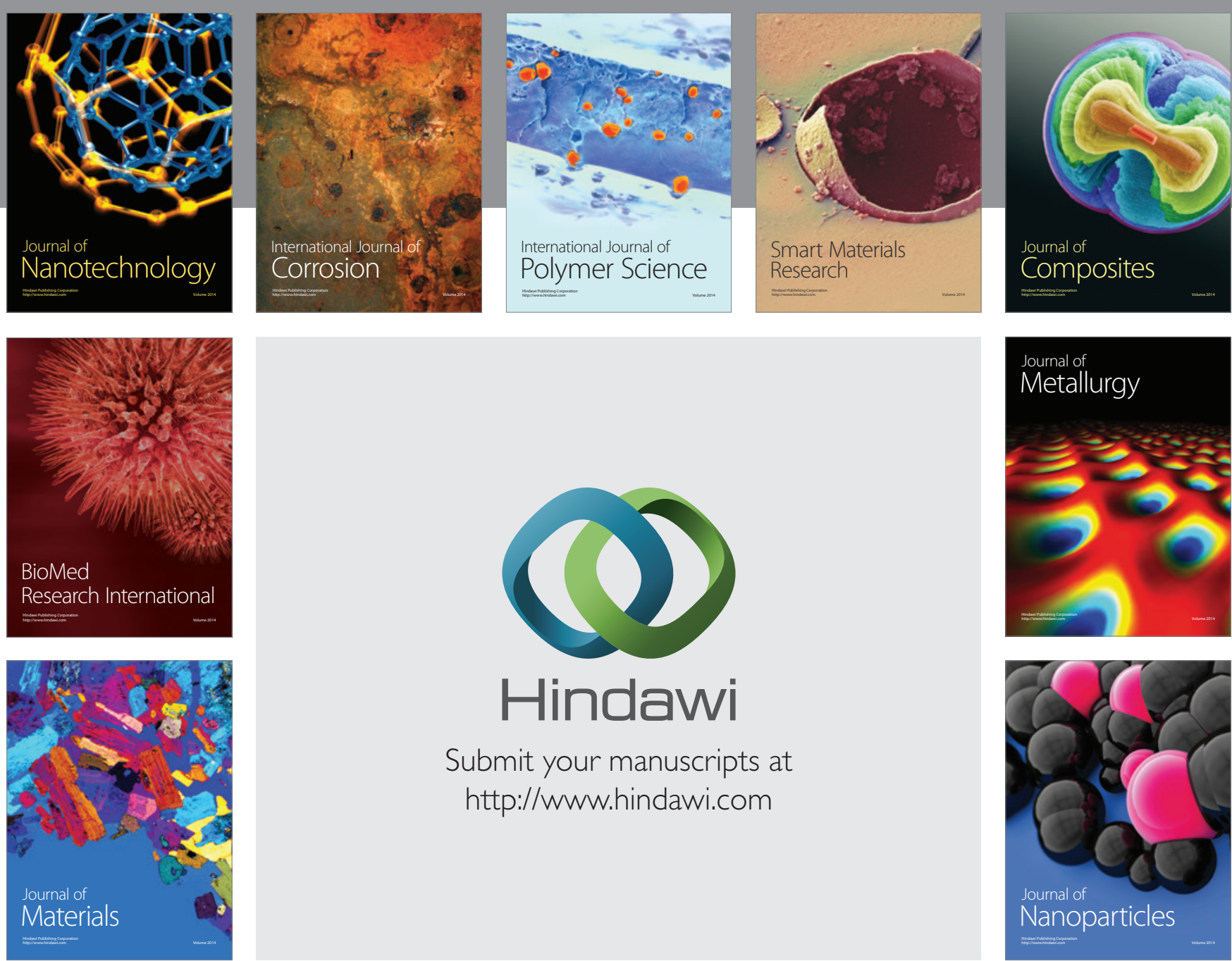

\section{Hindawi}

Submit your manuscripts at

http://www.hindawi.com

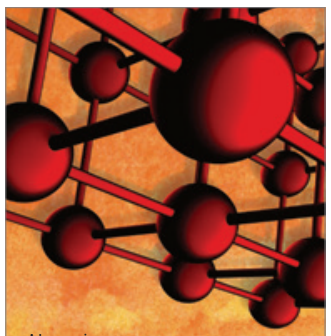

Materials Science and Engineering
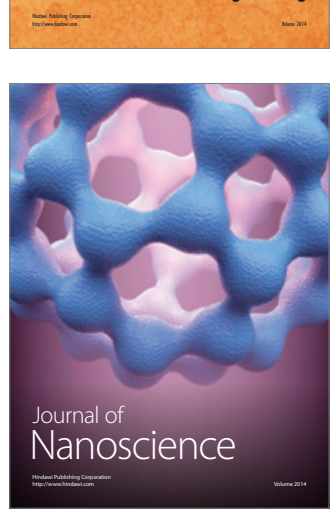
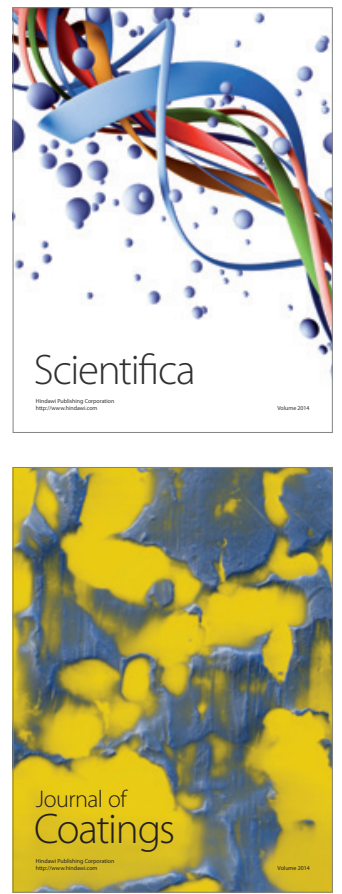
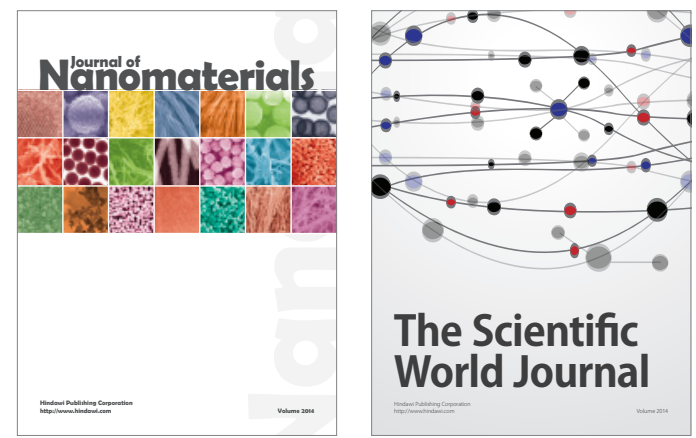

The Scientific World Journal
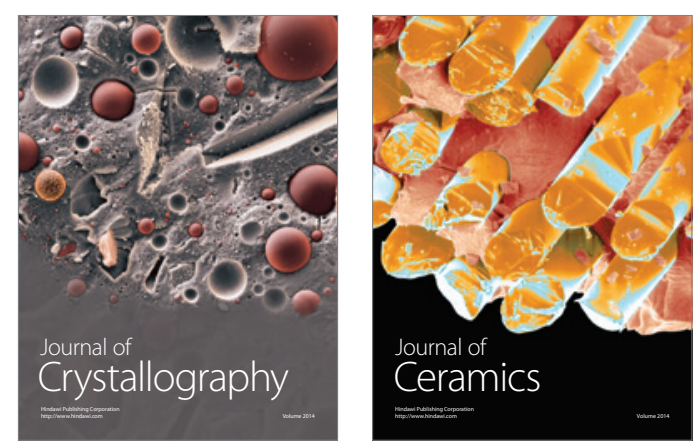
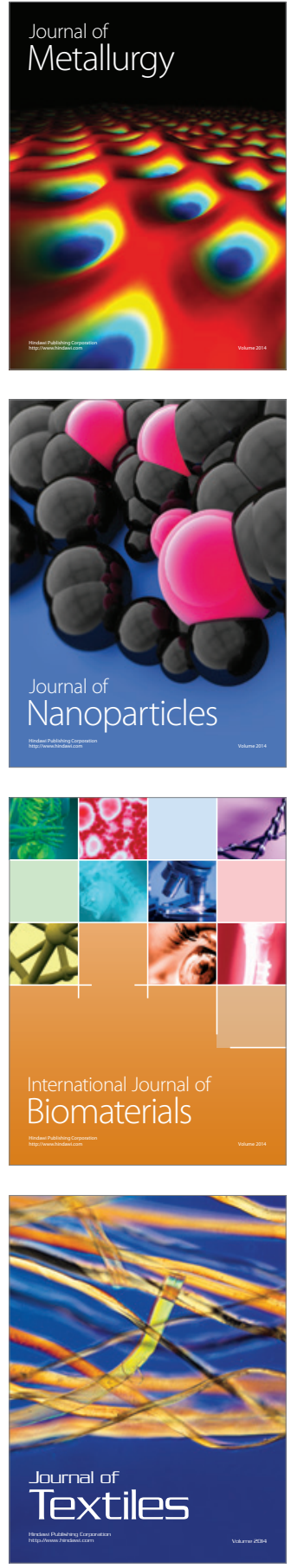